\title{
Ventriculoarterial decoupling in human septic shock
}

\author{
Fabio Guarracino ${ }^{1 *}$, Baldassare Ferro ${ }^{1}$, Andrea Morelli ${ }^{2}$, Pietro Bertini ${ }^{1}$, Rubia Baldassarri ${ }^{1}$ and Michael R Pinsky ${ }^{3}$
}

\begin{abstract}
Introduction: Septic shock is the most severe manifestation of sepsis. It is characterized as a hypotensive cardiovascular state associated with multiorgan dysfunction and metabolic disturbances. Management of septic shock is targeted at preserving adequate organ perfusion pressure without precipitating pulmonary edema or massive volume overload. Cardiac dysfunction often occurs in septic shock patients and can significantly affect outcomes. One physiologic approach to detect the interaction between the heart and the circulation when both are affected is to examine ventriculoarterial coupling, which is defined by the ratio of arterial elastance (Ea) to left ventricular end-systolic elastance (Ees). In this study, we analyzed ventriculoarterial coupling in a cohort of patients admitted to ICUs who presented with vs without septic shock.
\end{abstract}

Methods: In this retrospective cross-sectional opportunity study, we measured routine hemodynamics using indwelling arterial and pulmonary arterial catheters and transthoracic echocardiograms in 25 septic patients (group S) and 25 non-septic shock patients (group C) upon ICU admission. Ees was measured by echocardiography using a single-beat (Eess $)_{S B}$ method. Ea was calculated as 0.9 systolic arterial pressure/stroke volume, and then the $\mathrm{Ea} / \mathrm{Ees}_{\mathrm{SB}}$ ratio was calculated (normal value $<1.36$ ).

Results: In group S, 21 patients had an Ea/Ees $S_{S B}$ ratio $>1.36$ (uncoupled). The four patients with Ea/Ees $S_{S B}$ ratios $\leq 1.36$ had higher Ees $S_{S B}$ values than patients with Ea/Ees $S_{S B}$ ratios $>1.36(P=0.007)$, although Ea measurements were similar in both groups $(P=0.4)$. In group $C$, five patients had uncoupled Ea/Ees $s_{B}$ ratios. No correlation was found between Ees $_{S B}$ and left ventricular ejection fraction and between Ea/Ees $s_{S B}$ ratio and mixed venous oxygen saturation in septic shock patients.

Conclusions: Upon admission to the ICU, patients in septic shock often display significant ventriculoarterial decoupling that is associated with impaired left ventricular performance. Because Ea/Ees decoupling alters cardiovascular efficiency and cardiac energetic requirements independently of Ea or Ees, we speculate that septic patients with ventriculoarterial uncoupling may benefit from therapy aimed at normalizing the Ea/Ees ratio.

\section{Introduction}

Septic shock is characterized in the resuscitated patient as a hyperdynamic, hypotensive cardiovascular state associated with multiorgan dysfunction and metabolic disturbances consistent with tissue dysoxia [1]. Importantly, peripheral vasodilation (relative hypovolemia and low systemic vascular resistance) can mask coexistent cardiac dysfunction [2,3]. Cardiac dysfunction in septic shock includes left ventricular (LV) diastolic and systolic

\footnotetext{
* Correspondence: Fabiodoc64@hotmail.com

${ }^{1}$ Department of Anesthesia and Critical Care Medicine, University Hospital of Pisa, Via Paradisa 2, Pisa 56124, Italy

Full list of author information is available at the end of the article
}

dysfunction secondary to primary myocardial injury or right ventricular dysfunction due to pulmonary hypertension [4]. The combined loss of peripheral vasomotor tone and cardiac contractile impairment may potentially result in ventriculoarterial decoupling, the consequences of which can be worsening cardiac energetics and performance.

Suga and Sagawa [5] and Sunagawa et al. [6] analyzed the circuit from the standpoint of LV ejection fraction (LVEF). In this model, LV contractility is described by the end-systolic pressure-volume relationship (ESPVR). The slope of the ESPVR, called end-systolic elastance (Ees), is a load-independent measure of cardiac contractility. End-systolic pressure is also a function of both LV

\section{() Biomed Central}


stroke volume (SV) and a characteristic of the arterial outflow tract. The greater the SV for a given vascular tone, the greater the systolic arterial pressure. Similarly, increases in arterial systolic pressure for a constant preload and Ees will decrease SV and increase end-systolic volume. The slope representing the relationship between $\mathrm{SV}$ and systolic arterial pressure as SV is varied is called arterial elastance (Ea) (Figure 1). Thus, SV is both limited and defines end-systolic pressure through arterioventricular coupling [6]. Maximal myocardial efficiency, defined as the amount of external work performed for myocardial oxygen consumed, occurs when Ea is approximately onehalf Ees [7] and has stronger dependence on Ea than on Ees [8]. Accordingly, the Ea/Ees ratio is a sensitive and independent estimate of cardiovascular efficiency [5]. A normally coupled human cardiovascular system has an $\mathrm{Ea} /$ Ees ratio $=1 \pm 0.36$ (median $\pm \mathrm{IQR}$ ), with normal values being $2.2 \pm 0.8 \mathrm{mmHg} / \mathrm{ml}$ for $\mathrm{Ea}$ and $2.3 \pm 1$ $\mathrm{mmHg} / \mathrm{ml}$ for Ees [9].

Uncoupling, defined as an Ea/Ees ratio $>1.36$, can result from changes in Ea, Ees or both. Uncoupling reflects a reduction in LV ejection efficiency, which can promote LV energetic failure. Although septic shock is associated with hypotension, which unloads LV ejection, it may, if unbalanced, result in Ea/Ees decoupling. We recently documented a primary decrease in peripheral impedance and an increase in peripheral compliance associated with a decrease in central aortic compliance in a porcine model of early endotoxic shock [10]. We hypothesized that similar Ea/Ees decoupling may also be present in

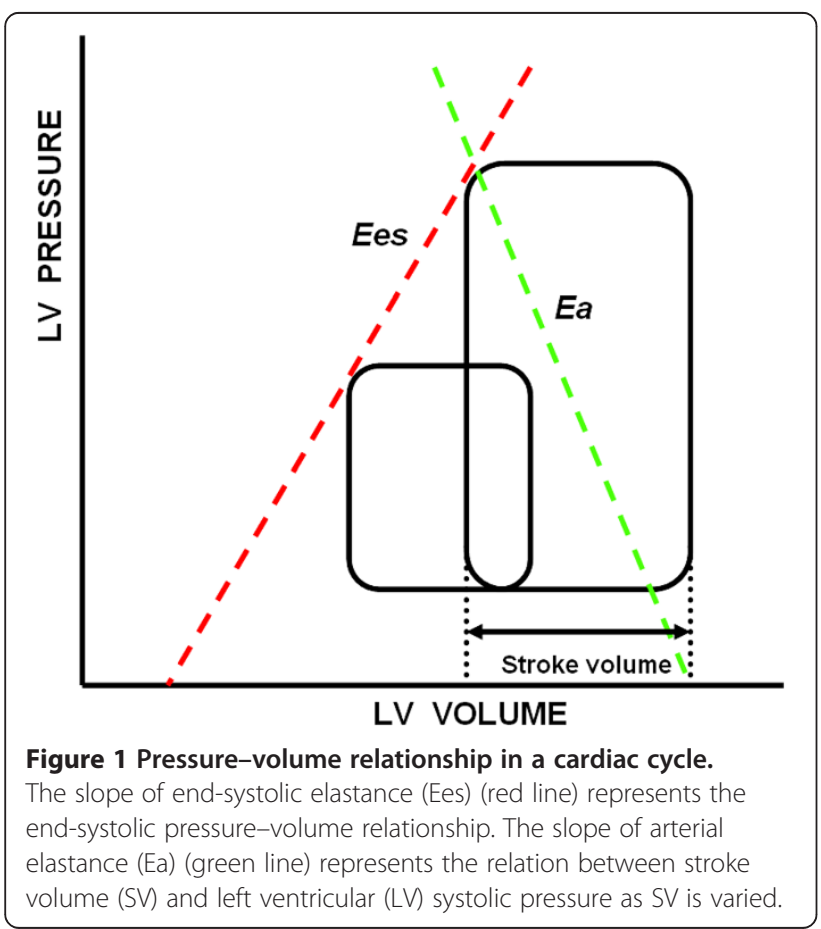

septic patients and that this uncoupling may contribute to the observed impaired LV ejection effectiveness.

Ea is a lumped parameter of arterial tone which is influenced by arterial resistance and compliance, aortic impedance and systolic and diastolic time intervals. It reflects the net arterial load on LV ejection. Because end-systolic pressure follows maximal ejection pressure and end-systole occurs as LV relaxation starts, endsystolic pressure can be approximated as $90 \%$ of systolic arterial pressure and SV [5,6]. Similarly, Ees is defined as the slope of the LV ESPVR derived from a series of LV end-systolic pressure-volume points created by rapidly varying preload or afterload such that intrinsic contractility remains constant [6]. We previously showed that end-inspiratory hold maneuvers rapidly decrease preload, thus allowing the measurement of Ees [11]. Still, the need to rapidly alter either preload or afterload and also use invasive LV catheterization to measure LV volume has kept the bedside measurement of Ees out of the realm of routine bedside practice.

The demonstration that Ees can be estimated in a single beat $\left(\mathrm{Ees}_{\mathrm{SB}}\right)$ [12], and the subsequent validation of a noninvasive echocardiographic method to measure Ees $_{\mathrm{SB}}$ [13] through the measure of LVEF, SV, pre-ejection time and systolic time interval when coupled with systolic and diastolic arterial pressure (Figure 2), made the clinical bedside measure possible. Thus, using these bedside estimates of Ea and Ees, we measured in patients presenting with vs without septic shock to analyze the presence of native ventriculoarterial decoupling.

\section{Methods}

After we obtained approval from the ethical committees for human biomedical research at both the University Hospital of Pisa and the University of Pittsburgh, we conducted a retrospective cross-sectional study between July 2011 and February 2013 to elaborate hemodynamic data obtained from (1) patients presenting with septic shock as defined by the international consensus conference definition and treated them following the Surviving Sepsis Campaign guidelines [14] and (2) patients admitted to the ICU without presumed septic shock. Patients with a history of cardiac disease and preoperative cardiac surgery patients were excluded. Informed consent was obtained from all patients, and all were studied immediately after initial fluid resuscitation in accordance with the Surviving Sepsis Campaign guidelines, but prior to starting therapy with any vasoactive pharmacological agents.

We analyzed a series of measured hemodynamic variables and calculated parameters at the time of diagnosis of septic shock in our septic shock cohort (8 women and 17 men, age $69 \pm 8$ years (median \pm IQR)) and at the time of ICU admission in our non-septic shock cohort 


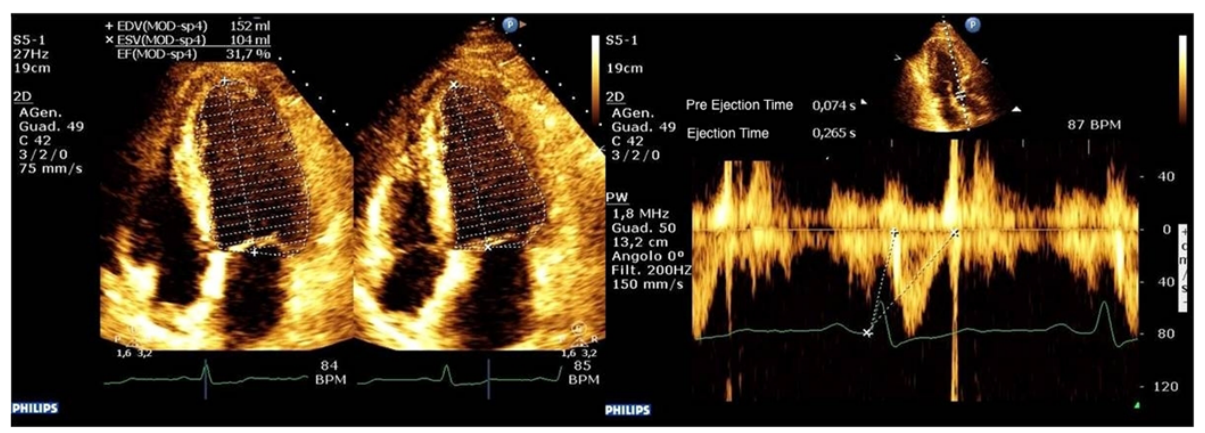

Figure 2 Left ventricular end-systolic elastance was calculated by using the single-beat method. These echocardiographic scans display the evaluation of ejection fraction (left image) and preejection and ejection time (right image) using aortic Doppler waveforms. Normalized ventricular elastance at arterial end-diastole (End) was measured according to the following formula:

$E_{e s(s b)}=\left[P_{d}-\left(E_{N d(e s t)} \times P_{s} \times 0.9\right)\right] /\left[S V \times E_{N d(e s t)}\right]$

$E_{N d(\text { avg })}=\Sigma a_{i} \times t_{N d}$

$i=0$

$E_{N d(\text { est })}=0.0275-0.165 \times E F+0.3656 \times\left(P_{d} / P_{e s}\right)+0.515 \times E_{N d(\text { avg })}$

where $a_{\mathrm{i}}$ values are $0.35695,-7.2266,74.249,-307.39,684.54 .-856.92,571.95$ and -159.1 for $i=1$ to $i=7$, respectively. The value $t_{\mathrm{Nd}}$ value was determined by the ratio of pre-ejection period (R-wave to flow onset) to total systolic period (R-wave to end-flow), with the time of onset and termination of flow-defined Doppler. Systolic blood pressure and diastolic blood pressure were measured invasively. The single-beat method used to calculate left ventricular end-systolic elastance was previously validated by Chen et al. [8].

with neurological problems but otherwise hemodynamically stable (11 women and 14 men, age $60 \pm 11$ years). These data included cardiac index (CI), heart rate and mean arterial pressure (MAP) invasively measured at a radial arterial site, as well as pulmonary artery occlusion pressure $\left(\mathrm{P}_{\text {pao }}\right)$ and mixed venous oxygen saturation $\left(\mathrm{SvO}_{2}\right)$ measured using a continuous cardiac output pulmonary artery catheter equipped with fiber optics (Swan-Ganz oximetry thermodilution catheter; Edwards Lifesciences, Irvine, CA, USA).

In nonseptic patients in whom a pulmonary artery catheter was not inserted at the discretion of the attending intensivist, the CI was measured using a pulse contour method (Vigileo Monitor/FloTrac Sensor System; Edwards Lifesciences), and central venous oxygen saturation $\left(\mathrm{ScvO}_{2}\right)$ was measured instead of $\mathrm{SvO}_{2}$. None of the nonseptic patients were receiving vasoactive drug therapy at the time they were examined.

All patients in both groups had a transthoracic echocardiographic examination upon admission, all of which were performed by the same operator. Echocardiography was performed with a CX50 ultrasound system and an S5-1 Sector Array Transducer (Koninklijke Philips Electronics NV, Eindhoven, the Netherlands).

Parameters calculated using data gathered from the echocardiographic examination included LVEF, SV, preejection time and systolic time. $\operatorname{Ees}_{\mathrm{SB}}$ was estimated by using the method of Chen et al. [13]. Ea was calculated as $0.9 \times$ (systolic arterial pressure/SV), and the $\mathrm{Ea} / \mathrm{Ees}_{\mathrm{SB}}$ ratio was then calculated.
Statistical data are expressed as median \pm IQR. Considering the small number of patients in our study, a nonparametric unpaired Mann-Whitney $U$ test was used to compare groups. Spearman's coefficient of rank correlation $(\rho)$ was used to correlate variables. Fisher's exact test was used in the analysis of contingency tables.

\section{Results}

The data derived from 50 patients were analyzed. The 25 patients with a diagnosis of septic shock presented in a hyperdynamic (median $\mathrm{CI}=2.77 \mathrm{~L} / \mathrm{min} / \mathrm{m}^{2}(\mathrm{IQR}=2.4$ to 3.8$)$ and $\mathrm{HR}=115$ beats $/ \mathrm{min}(\mathrm{IQR}=109$ to 124$)$, hypotensive (MAP 58 IR 53-60) $\mathrm{mmHg}$ ) state with a median $\mathrm{P}_{\text {pao }}$ of $13 \mathrm{mmHg}$ (IQR $=10$ to 14.2$)$, median LVEF of $40 \%$ (IQR $=32.25$ to 51.5$), \mathrm{SvO}_{2}$ of $60 \%$ (IQR = 58 to 69 ) and an $\mathrm{Ea} / \mathrm{Ees}_{\mathrm{SB}}$ ratio of 1.81 (IQR $=1.49$ to 2.02) following initial fluid resuscitation. Only four patients had an $\mathrm{Ea} / \mathrm{Ees}_{\mathrm{SB}}$ ratio $\leq 1.36$ at the time of diagnosis of septic shock, whereas the remaining twenty-one patients had an $\mathrm{Ea} / \mathrm{Ees}_{\mathrm{SB}}$ ratio $>1.36$ (Figure 3). The four patients with $\mathrm{Ea} / \mathrm{Ees}_{\mathrm{SB}}$ ratios $\leq 1.36$ had higher $\mathrm{Ees}_{\mathrm{SB}}$ compared with twenty-one patients with $\mathrm{Ea} / \mathrm{Ees}_{\mathrm{SB}} \mathrm{ra-}$ tios $>1.36(P=0.007)$, though Ea was similar in both the coupled and uncoupled septic shock patients $(P=0.4)$. We found no correlation between $\operatorname{Ees}_{\mathrm{SB}}$ and $\operatorname{LVEF}(\rho=-0.0809$, $P=0.7007)$ or between $\mathrm{Ea} / \mathrm{Ees}_{\mathrm{SB}}$ ratio and $\mathrm{SvO}_{2}$ in septic shock patients $(\rho=-0.293, P=0.15)$.

The 25 patients admitted in a non-septic shock state presented with a normal systemic flow state $(\mathrm{CI}=2.8 \mathrm{~L} /$ $\mathrm{min} / \mathrm{m}^{2}(\mathrm{IQR}=2.6$ to 3$\left.)\right) ; \mathrm{HR}=80$ beats $/ \mathrm{min}(\mathrm{IQR}=71$ 

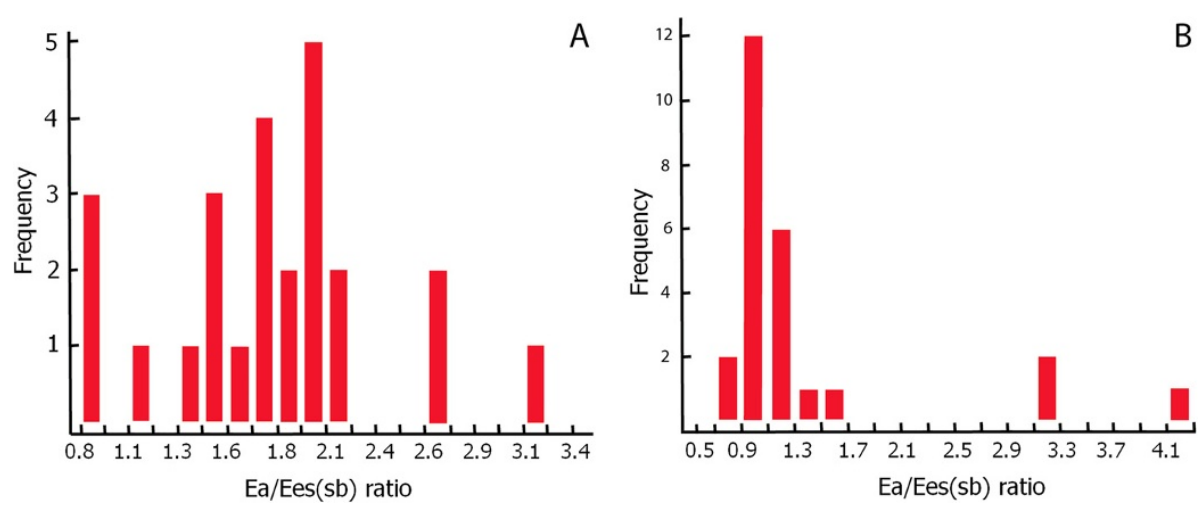

Figure 3 Graphs representing the distribution of ratios of arterial elastance to single-beat end-systolic elastance in newly diagnosed septic shock patients (A) and non-septic shock patients (B).

to $104 ; \mathrm{MAP}=70 \mathrm{mmHg}(\mathrm{IQR}=67$ to 71$)$ with an $\mathrm{LVEF}$ of $54 \%(\mathrm{IQR}=47.5$ to 58$), \mathrm{ScvO}_{2}=62 \%(\mathrm{IQR}=56.8$ to $69)$ and $\mathrm{Ea} / \mathrm{Ees}_{\mathrm{SB}}$ ratio $=1.07(\mathrm{IQR}=0.95$ to 1.14$)$. Five of these patients had a calculated $\mathrm{Ea} / \mathrm{Ees}_{\mathrm{SB}}$ ratio $>1.36$, and the remaining twenty patients had $\mathrm{Ea} / \mathrm{Ees}_{\mathrm{SB}}$ ratios $<1.36$ (Figure 3 ). Table 1 shows comparisons of variables between septic and nonseptic patients. Compared with non-septic shock patients, septic shock patients had reduced $\mathrm{Ea}$; lower $\mathrm{Ees}_{\mathrm{SB}}$, LVEF and MAP; and higher $\mathrm{Ea} / \mathrm{Ees}_{\mathrm{SB}}$ ratios and HRs. Table 2 shows the absence of correlation between $\mathrm{Ea} / \mathrm{Ees}_{\mathrm{SB}}$ status and normal or reduced LVEF in septic shock patients $(P=0.5)$ in a $2 \times 2$ contingency table.

\section{Discussion}

Our data show that, using bedside monitoring techniques [15], most of our patients who presented to ICUs in septic shock had significant ventriculoarterial decoupling independent of the commonly seen reduced Ea, whereas our non-septic shock patients displayed preserved ventriculoarterial coupling. This altered ventriculoarterial coupling in septic patients occurred despite a preserved or even elevated CI. These data suggest that, in septic shock patients, decoupling was associated with the observed impaired LV performance and reduced Ea. The lack of correlation between LVEF and $\operatorname{Ees}_{\mathrm{SB}}$ suggests that LVEF cannot be considered a clinical index of contractility or ventricular performance. This conclusion is not surprising, because LVEF is a function not only of contractility but also of Ea. On the basis of these data, we speculate that the use of vasoconstrictors in septic shock with the aim of maintaining sufficient MAP according to the published Surviving Sepsis Campaign guidelines could be the cause of the reduced LVEF, as previously suggested [3].

Ventriculoarterial decoupling is an important index of cardiovascular inefficiency. An adequate Ea/Ees ratio is fundamental for efficient cardiovascular performance and is a determinant of cardiac energetics [16]. Ea/Ees ratio reflects the interaction between cardiac function and the arterial system that is necessary to modulate the cardiovascular response to either physiological or pathological conditions. The changes in $\mathrm{CO}$ and vascular resistance in different physiological conditions (for example, age, exercise, rest) and pathological conditions (for example, hypertension, heart failure, diabetes) strictly depend on both LV function and the arterial system. Aging and cardiovascular diseases such as hypertension,

Table 1 Comparison of hemodynamic variables between septic shock and non-septic shock patients ${ }^{a}$

\begin{tabular}{|c|c|c|c|}
\hline Measurements & Septic shock patients & Non-septic shock patients & $P$-value \\
\hline $\mathrm{Cl}, \mathrm{L} / \mathrm{min} / \mathrm{m}^{2}$ & 2.7 (2.4 to 3.8$)$ & 2.8 (2.6 to 3 ) & 0.76 \\
\hline HR, beats/min & 115 (109 to 124$)$ & 80 (71 to 104$)$ & $<0.0001$ \\
\hline $\mathrm{SAP}, \mathrm{mmHg}$ & 85 (75 to 92) & 120 (95 to 135$)$ & $<0,0001$ \\
\hline $\mathrm{MAP}, \mathrm{mmHg}$ & $58(53$ to 60$)$ & $70(67.8$ to 71.3$)$ & $<0.0001$ \\
\hline LVEF, \% & 40 (32 to 52 ) & 54 (48 to 58 ) & 0.0098 \\
\hline $\mathrm{Ees}_{\mathrm{SB}}, \mathrm{mmHg} / \mathrm{ml}$ & 0.7 (0.59 to 1.1$)$ & $2.1(1.57$ to 2.3$)$ & $<0.0001$ \\
\hline $\mathrm{Ea}, \mathrm{mmHg} / \mathrm{ml}$ & $1.4(1.1$ to 1.48$)$ & 2.3 (2.02 to 2.45$)$ & $<0.0001$ \\
\hline $\mathrm{Ea} \mathrm{Ees}_{\mathrm{SB}}$ ratio & 1.81 (1.49 to 2.03$)$ & 1.07 (0.95 to 1.18$)$ & 0.01 \\
\hline
\end{tabular}

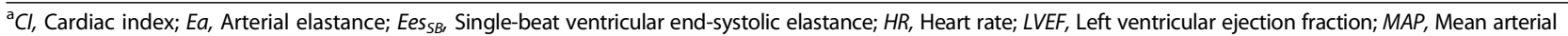
pressure; SAP, Systolic arterial pressure. Data are expressed as median and IQR. $P<0.05$ was considered statistically significant. 
Table 2 Analysis of correlation between ventriculoarterial coupling and left ventricular ejection fraction in septic shock patients ${ }^{a}$

\begin{tabular}{lll}
\hline & $\begin{array}{l}\text { LVEF normal } \\
(\geq 50 \%)\end{array}$ & $\begin{array}{l}\text { LVEF reduced } \\
(<50 \%)\end{array}$ \\
\hline Ea/Ees $_{\mathrm{SB}}$ normal $(<1.36), n$ & 2 & 2 \\
Ea $/$ Ees $_{\mathrm{SB}}$ altered $(>1.36), n$ & 6 & 15
\end{tabular}

${ }^{a} E a$, Arterial elastance; $E a / E s_{S B}$, Single-beat ventriculoarterial coupling; Ees, End-systolic elastance; $L V E F$, Left ventricular ejection fraction. $P=0.5$ by Fisher's exact test.

coronary artery disease, congestive heart failure and cardiac valve dysfunction can alter $\mathrm{Ea} /$ Ees coupling by reducing $\mathrm{LV}$ performance, increasing Ea or both [17].

Many researchers have shown that vasoactive drugs can affect ventriculoarterial coupling in several different clinical situations. The phosphodiesterase inhibitor E-1020 was found to increase heart mechanical efficiency by improving $\mathrm{Ea} /$ Ees coupling in heart disease patients [18]. Levosimendan, an inodilator, restored $\mathrm{Ea} /$ Ees coupling in patients with ischemic cardiomyopathy undergoing cardiac surgery [19], and enoximone improved $\mathrm{Ea} /$ Ees better than dobutamine in subjects with dilated cardiomyopathy [20,21]. Recently, Martin et al. demonstrated a better prognosis in patients with polytrauma using LV stroke work and ventriculoarterial coupling as targets of therapy [22].

Our study has some limitations. First, it is a retrospective analysis of prospectively collected data. Still, the data were collected in a consistent fashion from all participants. Second, the method we applied to measure Ees does not take into consideration the curvilinear shape of the elastance curve, which could be misleading if contractility was much reduced. However, estimated Ees values for our septic cohort were not depressed enough to reach this region of curvilinearity. Third, the definition of Ea was simplified as $\mathrm{Ea}=$ end-systolic pressure/SV, but this is routinely used as a valid surrogate. Furthermore, we measured arterial pressure from indwelling radial artery catheters that may either over- or underrepresent central aortic systolic pressure in septic patients. Differences of about $10 \mathrm{mmHg}$ may exist between peripheral and central pressure sites. However, the mathematical effect of systolic arterial pressure on the calculated $\mathrm{Ea} / \mathrm{Ees}_{\mathrm{SB}}$ would be small because it is used in the calculation of both parameters. Fourth, we did not reanalyze our septic patients following restoration of arterial pressure with vasopressor agents or after recovery to see if the associated uncoupling was resolved. This latter limitation forms the basis of an ongoing clinical study.

We cannot deduce the clinical implications of this decoupling from our analysis; however, because most of our septic shock patients had uncoupled Ea/Ees at the time of diagnosis, we speculate that patients with septic shock and decoupled $\mathrm{Ea} /$ Ees would benefit from vasoactive therapies aimed at normalizing the Ea/Ees ratio. For example, in a murine model of sepsis, Ducrocq et al. [23] demonstrated that increasing MAP by the use of selective $\alpha$-adrenergic vasopressors such as phenylephrine will unmask LV failure. Use of a more balanced $\alpha$ - and $\beta$-adrenergic agent, such as norepinephrine, may result in a better cardiovascular state with less potential for cardiac decompensation. Furthermore, the Surviving Sepsis Campaign guidelines suggest using inotropes as the last step in enhancing $\mathrm{SvO}_{2}$ values $>70 \%$ [14]. We did not find a correlation between $\mathrm{SvO}_{2}$ values and ventriculoarterial coupling in our septic shock patients, which is not surprising, because, owing to possible alterations in peripheral oxygen uptake, high venous oximetry cannot be considered a good index of perfusion or cardiovascular performance in these patients.

\section{Conclusion}

In septic shock patients, there is a higher percentage of ventriculoarterial decoupling compared to nonseptic patients admitted to ICUs. This decoupling is associated with impaired LV performance. Because ventriculoarterial decoupling is an index of cardiovascular inefficiency and a determinant of cardiac energetics, we speculate that such "uncoupled" patients may benefit from therapies aimed at normalizing the Ea/Ees ratio. However, this hypothesis remains to be tested.

\section{Key messages}

- Ea and LV Ees can be measured at the bedside in critically ill patients.

- Septic shock affects both ventricular and arterial elastance.

- Disproportionate changes in either ventricular or arterial elastance will lead to ventriculoarterial decoupling.

- Therapies aimed at normalizing the Ea/Ees ratio may improve cardiovascular efficiency.

\section{Abbreviations}

Cl: Cardiac index; CO: Cardiac output; Ea: Arterial elastance; Ees ${ }_{S B} E S:$ Single-beat end-systolic left ventricular elastance; Ees: End-systolic left ventricular elastance; EF: Ejection fraction; ESP: End-systolic pressure; ESPVR: End systolic pressurevolume relationship; HR: Heart rate; LV: Left ventricular; MAP: Mean arterial pressure; $\mathrm{P}_{\text {pao: }}$ Pulmonary artery occlusion pressure; $\mathrm{SCVO}_{2}$ : Central venous oxygen saturation; SV: Stroke volume; $\mathrm{SvO}_{2}$ : Mixed venous oxygen saturation.

\section{Competing interests}

The authors declare that they have no competing interests.

\section{Authors' contributions}

FG made substantial contributions to the conception and design of the work, interpretation of data and drafting the manuscript and revising it critically for important intellectual content. BF Acquired, analyzed and interpreted the data and drafted the manuscript. AM made substantial contributions to the design of the work and revised the manuscript critically 
for important intellectual content. PB made substantial contributions to the conception or design of the work; acquired, analyzed and interpreted the data; and drafted the manuscript. RB made substantial contributions to the conception and design of the work and drafted the manuscript. MP Interpreted the data and revised the manuscript critically for important intellectual content. All authors read and approved the final manuscript.

\section{Acknowledgements}

We thank all the ICU physicians for their contributions in designing the study and interpreting the data, as well as all the nurses for their generous cooperation.

\section{Author details}

${ }^{1}$ Department of Anesthesia and Critical Care Medicine, University Hospital of Pisa, Via Paradisa 2, Pisa 56124, Italy. ${ }^{2}$ Department of Anesthesiology and Intensive Care, University of Rome, "La Sapienza", Viale del Policlinico 155, Rome 00161, Italy. ${ }^{3}$ Department of Critical Care Medicine, University of Pittsburgh, 606 Scaife Hall, 3550 Terrace St, Pittsburgh, PA 15213, USA.

Received: 23 November 2013 Accepted: 3 April 2014

Published: 24 April 2014

\section{References}

1. Werdan $\mathrm{K}$, Müller-Werdan U: Elucidating molecular mechanisms of septic cardiomyopathy-the cardiomyocyte model. Mol Cell Biochem 1996, 163-164:291-303.

2. Flierl MA, Rittirsch D, Huber-Lang MS, Sarma JV, Ward PA: Molecular events in the cardiomyopathy of sepsis. Mol Med 2008, 14:327-336.

3. Vieillard-Baron A: Septic cardiomyopathy. Ann Intensive Care 2011, 1:6

4. Parker MM, McCarthy KE, Ognibene FP, Parrillo JE: Right ventricular dysfunction and dilatation, similar to left ventricular changes, characterize the cardiac depression of septic shock in humans. Chest 1990, 97:126-131.

5. Sunagawa K, Maughan WL, Sagawa K: Optimal arterial resistance for the maximal stroke work studied in isolated canine left ventricle. Circ Res 1985, 56:586-595.

6. Sunagawa K, Maughan WL, Burkhoff D, Sagawa K: Left ventricular interaction with arterial load studied in isolated canine ventricle. Am J Physiol 1983, 245:H773-H780.

7. Burkhoff $D$, Sagawa $K$ : Ventricular efficiency predicted by an analytical model. Am J Physiol 1986, 250:R1021-R1027.

8. Starling MR: Left ventricular-arterial coupling relations in the normal human heart. Am Heart J 1993, 125:1659-1666.

9. Chantler PD, Lakatta EG, Najjar SS: Arterial-ventricular coupling: mechanistic insights into cardiovascular performance at rest and during exercise. J Appl Physiol 2008, 105:1342-1351. A published erratum appears in J Appl Physiol 2009, 106:1027.

10. Hatib F, Jansen JRC, Pinsky MR: Peripheral vascular decoupling in porcine endotoxic shock. J Appl Physiol 2011, 111:853-860.

11. Kim HK, Alhammouri MT, Mokhtar YM, Pinsky MR: Estimating left ventricular contractility using inspiratory-hold maneuvers. Intensive Care Med 2007, 33:181-189.

12. Takeuchi M, Igarashi Y, Tomimoto S, Odake M, Hayashi T, Tsukamoto T, Hata K, Takaoka H, Fukuzaki H: Single-beat estimation of the slope of the end-systolic pressure-volume relation in the human left ventricle. Circulation 1991, 83:202-212.

13. Chen CH, Fetics B, Nevo E, Rochitte CE, Chiou KR, Ding PA, Kawaguchi M, Kass DA: Noninvasive single-beat determination of left ventricular end-systolic elastance in humans. J Am Coll Cardiol 2001, 38:2028-2034.

14. Dellinger RP, Levy MM, Rhodes A, Annane D, Gerlach H, Opal SM, Sevransky JE, Sprung CL, Douglas IS, Jaeschke R, Osborn TM, Nunnally ME, Townsend SR, Reinhart K, Kleinpell RM, Angus DC, Deutschman CS, Machado FR, Rubenfeld GD, Webb SA, Beale RJ, Vincent IL, Moreno R, Surviving Sepsis Campaign Guidelines Committee including the Pediatric Subgroup: Surviving Sepsis Campaign: international guidelines for management of severe sepsis and septic shock: 2012. Crit Care Med 2013, 41:580-637.

15. Guarracino F, Baldassarri R, Pinsky MR: Ventriculo-arterial decoupling in acutely altered hemodynamic states. Crit Care 2013, 17:213.

16. Prabhu SD: Altered left ventricular-arterial coupling precedes pump dysfunction in early heart failure. Heart Vessels 2007, 22:170-177.
17. Goto Y, Futaki S, Kawaguchi O, Hata K, Takasago T, Saeki A, Nishioka T, Suga H: Left ventricular contractility and energetic cost in disease models - an approach from the pressure-volume diagram. Jpn Circ J 1992, 56:716-721.

18. Takaoka H, Takeuchi M, Odake M, Hayashi Y, Mori M, Hata K, Yokoyama M: Comparison of the effects on arterial-ventricular coupling between phosphodiesterase inhibitor and dobutamine in the diseased human heart. J Am Coll Cardiol 1993, 22:598-606.

19. Guarracino F, Cariello C, Danella A, Doroni L, Lapolla F, Stefani M, Baldassarri $\mathrm{R}$, Vullo C: Effect of levosimendan on ventriculo-arterial coupling in patients with ischemic cardiomyopathy. Acta Anaesthesiol Scand 2007, 51:1217-1224.

20. Ishihara H, Yokota M, Sobue T, Saito H: Relation between ventriculoarterial coupling and myocardial energetics in patients with idiopathic dilated cardiomyopathy. J Am Coll Cardiol 1994, 23:406-416.

21. Beanlands RS, Bach DS, Raylman R, Armstrong WF, Wilson V, Montieth M, Moore CK, Bates E, Schwaiger M: Acute effects of dobutamine on myocardial oxygen consumption and cardiac efficiency measured using carbon-11 acetate kinetics in patients with dilated cardiomyopathy. J Am Coll Cardiol 1993, 22:1389-1398.

22. Martin RS, Norris PR, Kilgo PD, Miller PR, Hoth JJ, Meredith JW, Chang MC, Morris JA Jr: Validation of stroke work and ventricular arterial coupling as markers of cardiovascular performance during resuscitation. J Trauma 2006, 60:930-935.

23. Ducrocq N, Kimmoun A, Furmaniuk A, Hekalo Z, Maskali F, Poussier S, Marie PY, Levy B: Comparison of equipressor doses of norepinephrine, epinephrine, and phenylephrine on septic myocardial dysfunction. Anesthesiology 2012, 116:1083-1091.

doi:10.1186/cc13842

Cite this article as: Guarracino et al:: Ventriculoarterial decoupling in human septic shock. Critical Care 2014 18:R80.

\section{Submit your next manuscript to BioMed Central and take full advantage of:}

- Convenient online submission

- Thorough peer review

- No space constraints or color figure charges

- Immediate publication on acceptance

- Inclusion in PubMed, CAS, Scopus and Google Scholar

- Research which is freely available for redistribution 